\title{
Mammographic Breast Density as a Predictive Factor for Pathological and Clinical Response to Neoadjuvant Chemotherapy in Breast Cancer Patients
}

\author{
YOMNA M. HEGAZY, M.Sc.*; ASHRAF F. BARAKAT, M.D.*; AMR M. EL-BADRY, M.D.** and \\ WALID A. EL-MORSY, M.D.* \\ The Departments of Oncology* and Radiology**, Faculty of Medicine, Tanta University
}

\begin{abstract}
Background: Breast cancer is the most frequent malignant disease in women. Neoadjuvant chemotherapy of breast cancer leads to high clinical response rates of $70 \%$ to $90 \%$. Breast density (BD) is one of the most important factor that affect pathological complete response. Low $\mathrm{BD}$ is associated with more likelihood of pCR. Similarly low MBD $(<25 \%)$ was associated with better progression free survival in locally advanced patients treated with neoadjuvant chemotherapy.

Aim of the Study: Is to evaluate the impact of mammographic breast density as a predictive factor for pathological and clinical response to neoadjuvant chemotherapy in breast cancer patients.

Patient and Method: The present study was carried out on 50 patients with confirmed diagnosis of invasive breast cancer and treated with neoadjuvant chemotherapy. Mammographic breast density was assessed for each patient and its impact on clinical and pathological response to treatment.

Results: Patients with low breast density were more likely to achieve pCR than patients with high breast density.

Conclusion: Patient with low breast density are more likely to achieve complete and partial response to neoadjuvant chemotherapy.

Other factors affect response to neoadjuvant chemotherapy as, age less than 40 , low body mass index, premenopausa women, tumor grade 1, no lymphovascular invasion, absence of multicenteric tumor are more likely to achieve complete and partial response.
\end{abstract}

Key Words: Breast density (BD) - Pathological complete response $(p C R)$.

\section{Introduction}

BREAST cancer is the second most frequent cancer worldwide and the first among women, excluding non-melanoma skin cancer [1].

Correspondence to: Dr. Yomna M. Hegazy, The Department of Oncology, Faculty of Medicine, Tanta University
Neoadjuvant chemotherapy was originally applied in locally advanced breast cancer patients with the aim of tumor downstaging to allow for surgical resection [2].

Neoadjuvant chemotherapy has some potential advantages; it treats the systemic micrometastatic disease from the beginning; reduces the tumor load; increases the rate of conservative surgery; allows the in vivo evaluation of sensitivity to chemotherapy and a rapid modification of the therapeutic regimen [3].

Evidence then emerged that induction of pathological complete response (pCR) is a predictive of long-term outcome with improved survival [4]

Several predictive factors for response to neoadjuvant chemotherapy have been studied such as: Breast density, age, body mass index, hormonal receptors.

Mammographic breast density refer to the tissue composition of the breast which consist of stromal and epithelial components (radiodense tissue), which appear white on mammogram and fatty tissue (radiolucent tissue) which appears black on mammogram [5].

\section{Patients and Methods}

This is a retrospective study including $50 \mathrm{pa}-$ tients with confirmed invasive breast cancer presented at Clinical Oncology and Nuclear Medicine Department, Tanta University Hospital, between January 2014 to December 2016.

\section{Method:}

Data are collected and analysed include history and clinical examination and Local examination 
of the breast to assess tumor size, consistency, shape, fixation to overlying or underlying structure, its site within the breast. The breast skin is evaluated for edema, erythema, ulceration or dimpling and assessment of the nipple as regard the color, erosion, retraction or displacement.

Baseline mammography was performed for all patients through visual assessment of screen films of two-view mammograms (craniocaudal and mediolateral oblique) of each breast already performed at the time of diagnosis.

Mammography and ultrasonography was done after the last cycle of chemotherapy to compare the findings with mammography done at the start of the treatment.

\section{Statistical analysis of data:}

Data were analyzed using IBM SPSS Statistics software package version 20. Categorical data were presented as number and percentage. To detect impact of different parameters on clinical and pathological response to neoadjuvant chemotherapy in breast cancer patients Univariate logistic regression analaysis was used.

Predictors which are significant in Univariate logistic regression analaysis were entered in multivariate logistic regression analaysis.

\section{Results}

Table (1) illustrates, with univariate logistic regression analysis there are statistically significant impact of each sociodemographic parameters on pathological complete response separately, as less than 40 years old patients were more likely to have pCR compared to those whose age more than 40 years old, $(\mathrm{OR}=38$ (.15-.96), $p=.04)$.

Also Menopausal state had statistically significant impact on pCR, with [OR=409 (.19-.89), $p=.024]$, as well as BMI as showed in (Table 1).

Table (2) illustrates, with univariate logistic regression analysis there are statistically significant impact of some a histopathological parameters on clinical and pathological response separately, as tumors with grade 1 , absence of multicentric with no lympphovascular invasion more likely to have response as $[\mathrm{OR}=.45(.205-.98), p=.047, . \mathrm{OR}=.370$ (.18-.76), $p=.007, \mathrm{OR}=.381(.17-.86), p=.02] \mathrm{re}-$ spectively. While tumor stage has no statistically significant impact on response the same like estrogen/progesterone status. In contrast the patients with positive HER 2 more likely to have response [OR=214 (.062-.74), $p=.015]$.

Table (3) illustrates, with univariate logistic regression analysis there are statistically significant impact of Chemotherapy Type parameters on clinical and pathological response as $p=.038$ while number of cycle has no impact on response).

Table (4) illustrates that by Univariate logistic regression analysis, the difference between low breast density and high had statistically significant impact on response, as the odds ratio is $[\mathrm{OR}=.31$ (.117-.620) and $p=.002]$.

Table (1): Pathological complete response (pCR) rate according to sociodemographic parameters.

\begin{tabular}{|c|c|c|c|c|c|c|}
\hline \multirow{3}{*}{ Parameters } & \multicolumn{4}{|c|}{$\mathrm{pCR}$} & \multirow{3}{*}{$\mathrm{OR}^{1}\left(95 \% \mathrm{CI}^{2}\right)$} & \multirow{3}{*}{$p$} \\
\hline & \multicolumn{2}{|c|}{ Response } & \multicolumn{2}{|c|}{ No response } & & \\
\hline & No. & $\%$ & No. & $\%$ & & \\
\hline \multicolumn{7}{|l|}{ Age: } \\
\hline$<40$ & 6 & 12 & 16 & 32 & $.38(.15-.96)$ & $.04 *$ \\
\hline$>40 \mathbf{a}$ & 6 & 12 & 22 & 44 & & \\
\hline \multicolumn{7}{|l|}{ Menopause: } \\
\hline Premenopause & 9 & 18 & 22 & 44 & $.409(.19-.89)$ & $.024 *$ \\
\hline Postmenopause b & 3 & 6 & 16 & 32 & & \\
\hline \multicolumn{7}{|l|}{$B M I:$} \\
\hline$(18-24.9)$ & 3 & 6 & 5 & 10 & $.6(.14-.2 .5)$ & $.045 *$ \\
\hline$(25-29.9)$ & 4 & 8 & 10 & 20 & $.4(.12-1.3)$ & \\
\hline$(30-39.9)$ & 5 & 10 & 16 & 32 & $.3(.11-.85)$ & \\
\hline$(>40)^{\mathbf{c}}$ & 0 & 0 & 7 & 14 & & \\
\hline
\end{tabular}


Table (2): Pathological complete response (pCR) rate according to histopathological Characteristics parameters.

\begin{tabular}{|c|c|c|c|c|c|c|}
\hline \multirow{3}{*}{ Parameters } & \multicolumn{4}{|c|}{$\mathrm{pCR}$} & \multirow{3}{*}{$\mathrm{OR}^{1}\left(95 \% \mathrm{CI}^{2}\right)$} & \multirow{3}{*}{$p$} \\
\hline & \multicolumn{2}{|c|}{ Response } & \multicolumn{2}{|c|}{ No response } & & \\
\hline & No. & $\%$ & No. & $\%$ & & \\
\hline \multicolumn{7}{|l|}{ Tumor grade: } \\
\hline Grade 1 & 9 & 18 & 20 & 40 & $.45(.205-.98)$ & $.047^{*}$ \\
\hline Grade2 a & 3 & 6 & 18 & 36 & & \\
\hline \multicolumn{7}{|c|}{ Multicentric tumours: } \\
\hline Yesb & 2 & 4 & 11 & 22 & $.370(.18-.76)$ & $.007 *$ \\
\hline No & 10 & 20 & 27 & 54 & & \\
\hline \multicolumn{7}{|l|}{ LVI: } \\
\hline Yes $\mathbf{c}$ & 4 & 8 & 17 & 34 & $.381(.17-.86)$ & $.02 *$ \\
\hline No & 8 & 16 & 21 & 42 & & \\
\hline \multicolumn{7}{|l|}{ Tumor stage: } \\
\hline Stage 1 & 1 & 2 & 1 & 2 & 1 & .067 \\
\hline Stage2 & 4 & 8 & 6 & 12 & .67 & \\
\hline Stage3A & 4 & 8 & 17 & 34 & .235 & \\
\hline Stage $3 B^{d}$ & 3 & 6 & 14 & 28 & & \\
\hline \multicolumn{7}{|l|}{$E R / P R$ status: } \\
\hline Positive & 9 & 18 & 23 & 46 & $.511(.199-2.2)$ & .37 \\
\hline Negative & 3 & 6 & 15 & 30 & & \\
\hline \multicolumn{7}{|l|}{ HER2: } \\
\hline Positive & 9 & 18 & 14 & 28 & $.214(.062-.74)$ & $.015^{*}$ \\
\hline Negativee & 3 & 6 & 24 & 48 & & \\
\hline
\end{tabular}

1: OR, Odds ratio. $\quad$ 2: CI, Confidence interval. $\quad \mathbf{a}, \mathbf{b}, \mathbf{c}, \mathbf{d}, \mathbf{e}$ : Reference groups.

Table (3): Pathological complete response (pCR) rate according to treatment modalities.

\begin{tabular}{|c|c|c|c|c|c|c|}
\hline \multirow{3}{*}{ Parameters } & \multicolumn{4}{|c|}{$\mathrm{pCR}$} & \multirow{3}{*}{$\mathrm{OR}^{1}\left(95 \% \mathrm{CI}^{2}\right)$} & \multirow{3}{*}{$p$} \\
\hline & \multicolumn{2}{|c|}{ Response } & \multicolumn{2}{|c|}{ No response } & & \\
\hline & No. & $\%$ & No. & $\%$ & & \\
\hline \multicolumn{7}{|l|}{ Chemotherapy Type: } \\
\hline Anthracycline \& & 10 & 20 & 23 & 46 & $43(.21-.913)$ & $.038 *$ \\
\hline Taxane & & & & & $2(.04-.913)$ & \\
\hline Anthracycline & 2 & 4 & 10 & 20 & & \\
\hline Taxanea & 0 & 0 & 5 & 10 & & \\
\hline \multicolumn{7}{|l|}{ NO. cycle: } \\
\hline$<6$ & 2 & 4 & 11 & 22 & $.49(.09-2.6)$ & .404 \\
\hline $6-8$ & 10 & 20 & 27 & 54 & & \\
\hline
\end{tabular}

Table (4): Pathological complete response (pCR) rate according to breast density.

\begin{tabular}{|c|c|c|c|c|c|c|}
\hline \multirow{3}{*}{ Parameters } & \multicolumn{4}{|c|}{$\mathrm{pCR}$} & \multirow{3}{*}{$\mathrm{OR}^{1}\left(95 \% \mathrm{CI}^{2}\right)$} & \multirow{3}{*}{$p$} \\
\hline & \multicolumn{2}{|c|}{ Response } & \multicolumn{2}{|c|}{ No response } & & \\
\hline & No. & $\%$ & No. & $\%$ & & \\
\hline \multicolumn{7}{|c|}{ Breast Density: } \\
\hline Low & 7 & 14 & 52 & 68.4 & $.31(.117-.620)$ & $.002 *$ \\
\hline Higha & 5 & 10 & 24 & 31.6 & & \\
\hline
\end{tabular}




\section{Discussion}

Neoadjuvant chemotherapy is the standard care for treatment of locally advanced and inoperable breast cancer [6].

The ideal outcome of NAC is the complete absence of residual invasive tumor cells within excised breast tissue following NAC, or pathological complete response (pCR), which strongly predicts favorable prognosis as compared with patients who experience partial or no response (non-pCR) [7] .

Neoadjuvant chemotherapy help for more rapid assessment of the efficacy of new therapeutic agents and early cessation of ineffective treatment $[8]$

In this study we aimed to evaluate the impact of mammographic breast density and other clinical and histopathological factors on clinical and pathological response rate to neoadjuvant chemotherapy in breast cancer patients.

In this study several factors affect the response to neoadjuvant chemotherapy.

In this study, $10 \%$ of patient achieve complete response, $66 \%$ achieve partial response, $14 \%$ of patients have stable disease, $10 \%$ of patients have progressive disease.

One of the factors that affect response to neoadjuvant chemotherapy is the breast density, in this study about $10 \%$ from patient with high breast density achieve response (complete and partial) while $14 \%$ of patient of low breast density achieve response.

In Elsamany S et al., study 241 patients involved in this study after receiveing neoadjuvant chemotherapy; Low BD patients were more likely to have pCR compared to those with high BD, however, the difference was of borderline significance $(30.5 \%$ vs $19.5 \%$ respectively, $\mathrm{OR}=1.8,95 \% \mathrm{CI}=0.98-3.3$, $p=0.056)$ [9] .

However, Castaneda et al., 2014 failed to show any correlation between mammographic BD and pCR [10].

This difference may be due to the difference in the number of the patient included in different studies and also the quantitative visual assessment of mammographic BD utilized in our study has some limitations. It lacks the accurate estimation of BD provided by computer-aided assessment in addition to the hazard of inter-observer variation in $\mathrm{BD}$ evaluation.

However, this method has some advantages. It is a simple method that can be easily implemented in the routine practice without requiring special software and it classifies patients into easily interpretable groups.

Other factors also affect response to neoadjuvant chemotherapy and achievement of clinical and pathological response such as: Age, menopause, BMI, tumor grade, stage, multicenteric tumor, lymphvascular invasion, hormonal receptor status and HER2, treatment regimen and number of cycles.

In our study patients with age less than 50 were more likely to have clinical and pathological response as compared to those whose age more than 50 years old $[\mathrm{OR}=.38(.15-.96), p=.04]$.

Young patients seem to achieve higher response rates after neoadjuvant chemotherapy. In the GeparTrio study, age was the only independent predictive factor for achieving a pCR in triple negative breast cancer [11].

Body mass index also affect response to neoadjuvant chemotherapy, in our study patient with low BMI are more liable to achieve clinical and pathological response. $(p=.045)$.

So higher BMI was associated with worse $\mathrm{pCR}$ to NAC. In addition, its association with worse overall survival suggests that greater attention should be focused on this risk factor to optimize the care of breast cancer patients [12]

This refers to the importance of keeping normal body weight to improve the outcome of therapy of breast cancer patients. This poor outcome is consistent with data reported by Dawood et al., who displayed worse survival outcome in obese and over-weight patients with locally advanced breast cancer compared to normal/underweight counterparts [13].

Also menopausal status play a role in response to neoadjuvant chemotherapy, in our study premenopausal women are more likely to achieve response as compared to postmenopausal group $(p=0.024)$.

This can be explained as higher level of oestradiol produced in postmenopausal women through aromatisation of androgens in the adipose tissues 
lead to worse response to neoadjuvant chemotherapy [14].

There are also other histopathological parameters that affect response to neoadjuvant chemotherapy such as, tumor grade, stage, lymphovascular invasion, multicenteric tumors.

In our study; there are statistically significant impact of some histopathological parameters on clinical and pathological response separately, as tumors with grade 1 , no multicentric with no LVI more likely to have response as [OR $=.45(.205-$ $.98), p=.047, \mathrm{OR}=.370(.18-.76), p=.007, \mathrm{OR}=.381$ $(17-.86), p=.02]$ respectively. While tumor stage has no statistically significant impact on pCR.

The current Dutch guidelines recommend NAC in patients with a tumor larger than $2 \mathrm{~cm}$ or smaller high-risk tumors with as main goal a safer and less extensive surgery [15].

Some studies have shown that lymphovascular invasion is associated with "chemoresistant" cancers [16] and that its absence on core biopsies is associated with pathological complete response (pCR) and improved survival [17].

Others found that LVI was a significant component of clinical, pathologic scores that predict response and survival in women receiving NAC [18].

Although the exact mechanism is unknown, LVI may represent an aggressive tumor or tumor environment that could portend a worse prognosis.

In our study we found that patients with multicenteric tumor were less liable to achieve response.

This data is supported by GeparTrio, GeparQuattro, and GeparQuinto trials including 6134 women with breast cancer. The patients were classified as having unifocal $(n=4,733,77.1 \%)$, multifocal $(n=820,13.4 \%)$, or multicentric $(n=581$, $9.5 \%)$ tumors.

The respective $\mathrm{pCR}$ rates were $19.4,16.5$, and $14.4 \%$ respectievely.

So pCR was obtained more in patients with unifocal tumor than multicentreic tumor [19]

Hormonal receptor status and HER2 status also affect response to neoadjuvant chemotherapy. In our study ER, PR have no statistically significant impact on response, however the patients with positive HER2 more likely to have complete and partial response than patients with HER2 negative [OR=.214 (.062-,74), $p=.015]$.

In Houssami $\mathrm{N}$ et al., study data were collected from NAC trials study the effect of hormonal receptor on $\mathrm{pCR}$; the highest rates of response was observed among patients with triple-negative or estrogen receptor (ER)-negative, human epidermal growth factor receptor-2 (HER2)-positive (ER-/ HER2+) cancers, while very low pCR rates are seen in patients with ER+/HER2-tumors [20]

Treatment modalities also affect response rate, in our study patients treated wih anthracycline and taxenes achieve response more than patients received anthracycline only or taxenes and cyclophophsamide.

New studies evaluated the therapeutic response of combination therapy with anthracycline-taxane versus anthracycline alone [21].

Combination chemotherapy regimens, whether simultaneous or sequential, may duplicate the pathologic complete response rate (pCR), reaching $30 \%$ in a population that includes initial stages [22].

\section{Conclusion:}

- Response to neoadjuvant chemotherapy is affected by several factors such as; demographic factors, histopathological factors and breast denisty. Patient with low breast density are more likely to achieve complete and partial response to neoadjuvant chemotherapy.

- Other factors affect response to neoadjuvant chemotherapy as, age less than 40 , low body mass index, premenopausal women, tumor grade 1 , no lymphovascuar invasion, no multicenteric tumor are more likely to achieve complete and partial response.

- Treatment modalities also affect complete and partial response, in our study patients treated with anthracycline and taxenes achieve response than patients received anthracycline only or taxenes only.

\section{Recommendation:}

- More studies are needed on larger number of patients to asees effect of breast density on neoadjuvant chemotherapy.

- Breast density should be assesd before start of treatment.

\section{Conflict of interest:}

The authors declare no conflict of interest. 


\section{References}

1- SIEGEL R., WARD E., BRAWLEY O., et al.: Cancer statistics: The impact of eliminating socioeconomic and racial disparities on premature cancer deaths. CA. Cancer J. Clin., 61 (4): 212- 236, 2011.

2- CLEATOR S., PARTON M. and DOWSETT M.: The biology of neoadjuvant chemotherapy for breast cancer. Endocr Relat Cancer, 9: 183e95, 2002.

3- TAGHIAN A., EL-GHAMRY M.N. and MERAJVER S.D.: Clinical features and management of locally advanced and inflammatory breast cancer. Sep-Dec., 2009.

4- RASTOGI P., ANDERSON S.J., BEAR H.D., et al.: Preoperative chemotherapy: Updates of National Surgical Adjuvant Breast and Bowel Project Protocols B-18 and B-27. Journal of Clinical Oncology: Official Journal of the American Society of Clinical Oncology, 26 (5): 778785,2008 .

5- MCCORMACK V.A. and dos SANTOS SILVA I.: Breast density an parenchymal patterns as markers of breast cancer risk: A meta analysis. Cancer Epidemiol Biomarkers Prev., 15 (6): 1159- 1169, 2006.

6- LIU S.V., MELSTROM L., YAO K., et al.: Neoadjuvant therapy for breast cancer. J. Surg Oncol., 101 (4): 283 291,2010

7- LUANGDILOK S., SAMARNTHAI N. and KORPHAISARN K.: Association between pathological complete response and outcome following neoadjuvant chemotherapy in locally advanced breast cancer patients. J. Breast. Cancer, 17: 376- 85, 2014.

8- SAHOO S., DABBS D.J. and BHARGAVA R.: Pathology of neoadjuvant response of breast carcinoma. In: Dabbs D.J. (ed.) Breast pathology (vol. 1). Philadelphia, P.A.: Elsevier Saunders, pp. 519-535, 2012.

9- ELSAMANY S.: Mammographic breast density: Predictive value for pathological response to neoadjuvant chemotherapy in breast cancer patients, Breast. Oct., 24 (5): 576-81, 2015.

10- CASTANEDA C.A., FLORES R., ROJAS K., et al.: Association between mammographic features and response to neoadjuvant chemotherapy in locally advanced breast carcinoma. Hematol. Oncol. Stem. Cell. Ther., 7 (4): 149 e56, 2014.

11- HUOBER J., VON MINCKWITZ G., DENKERT C., et al.: Effect of neoadjuvant anthracycline-taxane-based chemotherapy in different biological breast cancer phenotypes: Overall results from the Gepar Trio study. Breast. Cancer. Res. Treat., 124: 133-140, 2010.
12- JENNIFER K. LITTON, ANA M. GONZALEZ-ANGULO, et al.: Brewster: Relationship Between Obesity and Pathologic Response to Neoadjuvant Chemotherapy Among Women With Operable Breast Cancer, 4072-4077, 2008.

13- DAWOOD S., BROGLIO K., GONZALEZ-ANGULO A.M., et al.: Prognostic value of body mass index in locally advanced breast cancer. Clin. Cancer. Res., 14: $1718 \mathrm{e} 25,2008$

14-GOODWIN P.J., ENNIS M., BAHL M., et al.: High insulin levels in newly diagnosed breast cancer patients reflect underlying insulin resistance and are associated with components of the insulin resistance syndrome. Breast. Cancer. Res. Treat., 114: 517e25, 2009.

15- NABON Mammacarcinoom Landelijke richtlijn, Versie: 2.0 (Dutch Breast Cancer Guideline), 2012.

16- UEMATSU T., KASAMI M., WATANABE J., et al.: Is lymphovascular invasion degree one of the important factors to predict neoadjuvant chemotherapy efficacy in breast cancer? Breast cancer (Tokyo, Japan), 18 (4): 309313, 2011.

17- KESKIN S., MUSLUMANOGLU M., SAIP P., et al.: Clinical and pathological features of breast cancer associated with the pathological complete response to anthracycline-based neoadjuvant chemotherapy. Oncology, 81 (1): 30-38, 2011.

18- ABDEL-FATAH T.M., BALL G., LEE A.H., et al.: Nottingham ClinicoPathological Response Index (NPRI) after neoadjuvant chemotherapy (Neo-ACT) accurately predicts clinical outcome in locally advanced breast cancer. Clinical cancer research: An official Journal of the American Association for Cancer Research, 21 (5): 1052-1062, 2015.

19- BEYHAN A.TASEVEN, M.D.: Impact of Multifocal or Multicentric Disease on Surgery and Locoregional, Distant and Overall Survival of 6,134 BreastCancer Patients Treated With Neoadjuvant Chemotherapy, Annuals of Surgical Oncology, Oct., 11, 2014.

20- HOUSSAMI N., MACASKILL P. and VON MINCKWITZ G.: Meta-analysis of the association of breast cancer subtype and pathologic complete response to neoadjuvant chemotherapy. Eur. J. Cancer, 48 (18): 3342-3354, 2012.

21- UNTCH M., MÖBUS V., KUHN W., et al.: Intensive dose-dense compared with conventionally scheduled preoperative chemotherapy for high-risk primary breast cancer. J. Clin. Oncol., 27 (18): 2938-2945, 2009.

22- SYMMANS W.F., PEINTINGER F., HATZIS C., et al.: Measurement of residual breast cancer burden to predict survival after neoadjuvant chemotherapy. J. Clin. Oncol., 25 (28): 4414- 4422, 2007. 


\section{كثافة الثلى بالماموجرام كعامل تنبؤى البواني

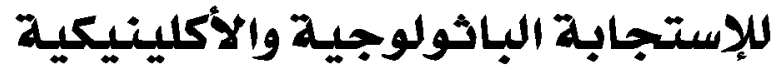

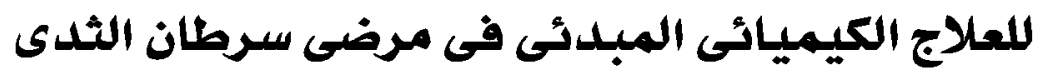

يعتبر سرطان الثدى من أكثر الأمراض أنتشاراً بين السيدات فى العالم كما تعتبر كثافة الثدى من أحد الاسباب المؤثرة فى الإصابة

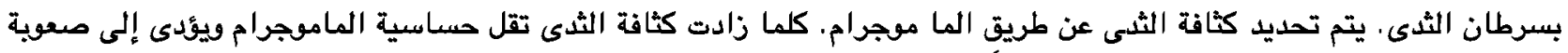

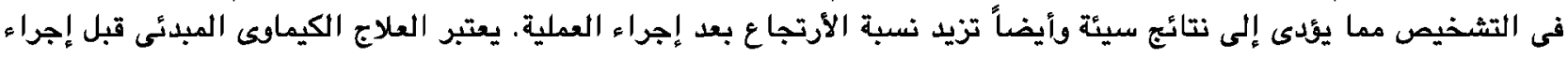

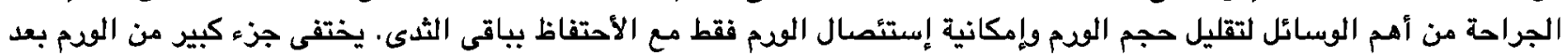

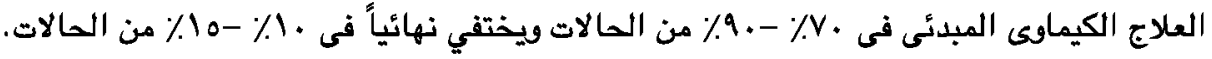

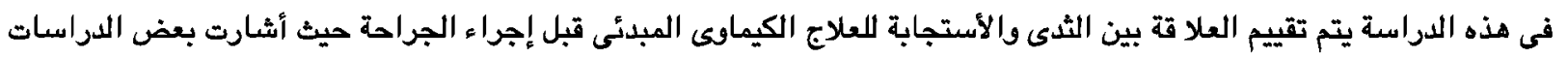

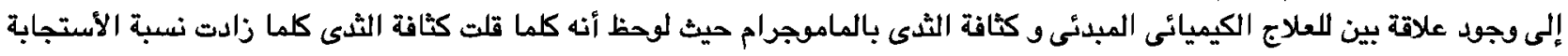
العلاج الكيميائى المبدئى. 